\title{
Synthesis of Planar Stiffness
}

\author{
J.M. Selig \\ School of Engineering \\ London South Bank University \\ seligjm@1sbu.ac.uk
}

\begin{abstract}
In this work the problem of designing systems of springs to achieve a desired stiffness matrix is considered. Only planar configurations are studied. After a brief section outlining the theory of the stiffness of planar systems the planar stiffness matrix of three typical design elements are found, simple springs, beams and pairs of stretched springs. A final section shows how arbitrary stiffness matrices can be achieved using three simple springs or two stretched spring pairs.
\end{abstract}

Keywords: Stiffness matrix, springs, beams.

\section{Introduction}

The synthesis of stiff or compliant systems is an important area of mechanism theory and robotics. There are many previous works in this area, see for example, [3, 4, 5]. Most of these concentrate on the problem of synthesising spatial stiffness matrices using so-called simple springs. These simple springs have zero natural length and are un-stretched. In this work the simpler problem of synthesising planar stiffnesses is considered. Using simple springs gives a mathematically interesting problem related to the secant varieties of a certain Veronese variety. More practical are simple beams and pairs of stretched springs, these are also considered.

\section{Planar Rigid-Body Geometry}

Here the basic geometric entities of points and lines are introduced and the action of the group of rigid-body displacement on these elements is described. As usual, given a coordinate frame, points can be represented as vectors,

$$
\vec{p}=\left(\begin{array}{c}
p_{x} \\
p_{y}
\end{array}\right),
$$

where $p_{x}$ and $p_{y}$ are the coordinates of the points in the fixed coordinate frame. It is often be more convenient to represent points using extended vectors,

$$
\tilde{p}=\left(\begin{array}{c}
\vec{p} \\
1
\end{array}\right)=\left(\begin{array}{c}
p_{x} \\
p_{y} \\
1
\end{array}\right)
$$


An advantage of this representation is that it extends easily to projective space $\mathbb{P}^{2}$. This space has homogeneous coordinates, $(x: y: w)$ and two sets of these homogeneous coordinates represent the same point in $\mathbb{P}^{2}$ if they are of the form $(x: y: w)$ and $(\lambda x: \lambda y: \lambda w)$ for some non-zero number $\lambda$. Thinking of points in the plane as points in the projective plane does however, introduce some extra points. The points with third homogeneous coordinate $w=0$ do not correspond to any points in the plane. They are usually referred to as points at infinity. In the projective plane these ideal points constitute a line.

The group $S E(2)$ acts by the familiar homogeneous representation on the extended points,

$$
\left(\begin{array}{l}
\vec{p} \\
1
\end{array}\right) \longmapsto\left(\begin{array}{cc}
R & \vec{t} \\
0 & 1
\end{array}\right)\left(\begin{array}{l}
\vec{p} \\
1
\end{array}\right)
$$

where, as usual,

$$
R=\left(\begin{array}{cc}
\cos \theta & -\sin \theta \\
\sin \theta & \cos \theta
\end{array}\right) \text { and } \vec{t}=\left(\begin{array}{c}
t_{x} \\
t_{y}
\end{array}\right)
$$

are respectively the rotation matrix and translation vector of the displacement.

In 2 dimensions lines are dual elements to points. The equation for a point to lie on a line is given by,

$$
n_{x} p_{x}+n_{y} p_{y}=d
$$

where $d$ is the perpendicular distance from the line to the origin of coordinates and $\vec{n}=\left(n_{x}, n_{y}\right)^{T}$ is the unit normal vector to the line. If lines are represented by 3 -vectors of the form,

$$
\ell=\left(\begin{array}{c}
\vec{n} \\
-d
\end{array}\right)=\left(\begin{array}{c}
n_{x} \\
n_{y} \\
-d
\end{array}\right),
$$

then the relation above can be represented by the pairing,

$$
\ell^{T} \tilde{p}=0
$$

That is, the point $\tilde{p}$ lies on the line $\ell$ if and only if $\ell^{T} \tilde{p}=0$.

Again, it is often useful to think of these as homogeneous coordinates of a $\mathbb{P}^{2}$. Notice that $\ell$ and $\lambda \ell$ give the same line if $\lambda$ is non-zero, although now $\lambda \vec{n}$ is no longer a unit vector and $\lambda d$ is not the perpendicular distance from the origin to the line. Thinking of lines as elements of a $\mathbb{P}^{2}$ means that the "line at infinity" must be included. This is the line with homogeneous coordinates $\ell_{\infty}=(0,0,1)^{T}$, on which the points at infinity $\tilde{p}=(x, y, 0)^{T}$, lie.

Thinking of lines as dual to points means that it is easy to see that lines transform under the inversetranspose representation of the group. That is the dual representation of the group,

$$
\left(\begin{array}{c}
\vec{n} \\
-d
\end{array}\right) \longmapsto\left(\begin{array}{cc}
R & 0 \\
-\vec{t}^{T} R & 1
\end{array}\right)\left(\begin{array}{c}
\vec{n} \\
-d
\end{array}\right)
$$

Since,

$$
\left(\begin{array}{cc}
R & \vec{t} \\
0 & 1
\end{array}\right)^{-T}=\left(\begin{array}{cc}
R & 0 \\
-\vec{t}^{T} R & 1
\end{array}\right)
$$

Twists are elements of the Lie algebra of the group, $S E(2)$ here. They can be thought of as infinitesimal rigid displacements. The Lie algebra of $S E(2)$ can be found from first princples or simply by abridging the 
results for the spatial case $S E(3)$, see [8]. So a general twist in the plane can be written as a 3-component vector,

$$
\mathbf{s}=\left(\begin{array}{c}
\omega \\
\vec{v}
\end{array}\right)=\left(\begin{array}{c}
\omega_{z} \\
v_{x} \\
v_{y}
\end{array}\right)
$$

where $\vec{v}$ is the linear velocity of a point instantaneously coincident with the origin and $\omega_{z}$ is the angular velocity of the body or lamina in this case. Under a rigid displacement these transform according to yet another representation of the group,

$$
\left(\begin{array}{c}
\omega \\
\vec{v}
\end{array}\right) \longmapsto\left(\begin{array}{cc}
1 & 0 \\
J \vec{t} & R
\end{array}\right)\left(\begin{array}{c}
\omega \\
\vec{v}
\end{array}\right), \quad \text { where } \quad J=\left(\begin{array}{cc}
0 & 1 \\
-1 & 0
\end{array}\right)
$$

Again, this can be found by computing the adjoint representation for $S E(2)$ or by looking at the adjoint representation of $S E(3)$ which is more widely known.

In the plane almost all twists are infinitesimal rotations about some point in the plane. So it is possible to associate points and twists. The mapping from twists to points is given by,

$$
\mathbf{s} \longmapsto \widetilde{L} \mathbf{s}=\left(\begin{array}{c}
J \vec{v} \\
\omega
\end{array}\right)=\tilde{p}, \quad \text { where } \quad \widetilde{L}=\left(\begin{array}{cc}
0 & J \\
1 & 0
\end{array}\right)=\left(\begin{array}{ccc}
0 & 0 & 1 \\
0 & -1 & 0 \\
1 & 0 & 0
\end{array}\right) .
$$

These matrices satisfy $J^{T}=J^{-1}=-J$ and $\widetilde{L}^{-1}=\widetilde{L}$.

Notice that, here points in the plane are represented by points in the projective space $\mathbb{P}^{2}$. Hence the centre of rotation of the twist $\mathbf{s}$ is given by the point $\vec{c}=\left(c_{x}, c_{y}\right)^{T}$ where,

$$
c_{x}=v_{y} / \omega, \quad \text { and } \quad c_{y}=-v_{x} / \omega .
$$

This shows that, when $\omega=0$ and the motion is an instantaneous translation, there is no finite centre of rotation. The extension of the map to the projective space $\mathbb{P}^{2}$ maps infinitesimal translations to points at infinity.

In 3 dimensions the combination of the torque and force acting on a rigid body is called a wrench. Wrenches are dual to twists, in 2-dimensions the abridged version of the wrench will be another 3component vector this time with the form,

$$
\mathbf{w}=\left(\begin{array}{l}
\tau \\
\vec{f}
\end{array}\right)=\left(\begin{array}{l}
\tau_{z} \\
f_{x} \\
f_{y}
\end{array}\right)
$$

where $\tau_{z}$ is the torque, and $f_{x}$ and $f_{y}$ the components of the force acting on the lamina. The duality is given by the pairing,

$$
\mathbf{w}^{T} \mathbf{s}=\tau_{z} \omega_{z}+f_{x} v_{x}+f_{y} v_{y}
$$

which gives the power, a scalar.

The effect of a rigid displacement on a wrench can be found from the duality. The dual representation is the inverse transpose of the representation for the twists, so given by,

$$
\left(\begin{array}{cc}
1 & 0 \\
J & R
\end{array}\right)^{-T}=\left(\begin{array}{cc}
1 & 0 \\
-R^{T} J \vec{t} & R^{T}
\end{array}\right)^{T}=\left(\begin{array}{cc}
1 & \vec{t}^{T} J R \\
0 & R
\end{array}\right)
$$


The action of a rigid displacement on a wrench is thus,

$$
\left(\begin{array}{l}
\tau \\
\vec{f}
\end{array}\right) \longmapsto\left(\begin{array}{cc}
1 & \vec{t}^{T} J R \\
0 & R
\end{array}\right)\left(\begin{array}{l}
\tau \\
\vec{f}
\end{array}\right)
$$

The line of action of the force gives a natural association between wrenches and lines. The line of action of a wrench $\mathbf{w}$ is given by the map,

$$
\mathbf{w} \longmapsto \widetilde{L}^{T} \mathbf{w}=\left(\begin{array}{c}
J \vec{f} \\
\tau
\end{array}\right)=\ell .
$$

Again this map is defined on projective space, non-zero constants multiplying every coordinate are irrelevant. The line associated with a pure torque is the line at infinity.

Notice that if a screw and a wrench are dual, that is if $\mathbf{w}^{T} \mathbf{s}=0$, then the point associated with the twist lies on the line associated to the wrench,

$$
\ell^{T} \tilde{p}=\mathbf{w}^{T} \widetilde{L} \widetilde{L} \mathbf{s}=\mathbf{w}^{T} \mathbf{s}=0,
$$

since, $\widetilde{L}^{2}=I$. This means that if the lamina is instantaneously rotating about a point on the axis of the applied force then no work is being done on the lamina.

\section{Stiffness Matrices}

Consider a lamina attached to a number of springs in the plane. Alternatively the lamina could be subject to some conservative force field. Stationary points of the potential energy correspond to equilibria of the lamina. At such an equilibrium configuration the Hessian matrix of the potential is the stiffness matrix of the configuration, see [6]. The Hessian is the matrix of second derivatives at the configuration and this gives an extended form of Hooke's law,

$$
\mathbf{w}=K \mathbf{s}
$$

where $\mathbf{w}$ is the wrench applied to the lamina, $\mathbf{s}$ is the resulting infinitesimal twist and $K$ the stiffness matrix. The stiffness matrix is a symmetric matrix and from physical considerations is also positive definite.

In the plane, all non-singular stiffness matrices are diagonaliseable. This is not the same as saying that any $3 \times 3$ symmetric matrix is diagonaliseable because we want the stiffness matrix to be diagonaliseable by rigid-body displacements, not by arbitrary non-singular matrices or even orthogonal matrices. So we need to know how the group $S E(2)$ acts on stiffness matrices. Since a stiffness matrix $K$, transforms a twist into a wrench the expression, $\mathbf{s}_{1}^{T} K \mathbf{s}_{2}$ is a scalar, so if the compliant element and the screws all undergo a rigid displacement or we just change the coordinates we are working in, this quantity must remain constant. This implies that under a rigid displacement the stiffness matrix $K$ will transform according to,

$$
K \longmapsto\left(\begin{array}{cc}
1 & 0 \\
J \vec{t} & R
\end{array}\right)^{-T} K\left(\begin{array}{cc}
1 & 0 \\
J \vec{t} & R
\end{array}\right)^{-1}=\left(\begin{array}{cc}
1 & \vec{t}^{T} J R \\
0 & R
\end{array}\right) K\left(\begin{array}{ccc}
1 & 0 \\
-R^{T} J \vec{t} & R^{T}
\end{array}\right)
$$

Writing the stiffness matrix in partitioned form,

$$
K=\left(\begin{array}{cc}
q & \vec{k}^{T} \\
\vec{k} & \Upsilon
\end{array}\right)
$$


a pure translation of $\vec{t}$ will give,

$$
K^{\prime}=\left(\begin{array}{cc}
1 & \vec{t}^{T} J \\
0 & I
\end{array}\right)\left(\begin{array}{cc}
q & \vec{k}^{T} \\
\vec{k} & \Upsilon
\end{array}\right)\left(\begin{array}{cc}
1 & 0 \\
-J \vec{t} & I
\end{array}\right)=\left(\begin{array}{cc}
q^{\prime} & \vec{k}^{T}+\vec{t}^{T} J \Upsilon \\
\vec{k}-\Upsilon J \vec{t} & \Upsilon
\end{array}\right),
$$

where $q^{\prime}=q-\vec{k}^{T} J \vec{t}+\vec{t}^{T} J \vec{k}-\vec{t}^{T} J \Upsilon J \vec{t}$. Choosing $\vec{t}=-J \Upsilon^{-1} \vec{k}$, the stiffness matrix becomes,

$$
K^{\prime}=\left(\begin{array}{cc}
q^{\prime} & \overrightarrow{0}^{T} \\
\overrightarrow{0} & \Upsilon
\end{array}\right)
$$

Notice that, with this choice for $\vec{t}$ we get $q^{\prime}=q-\vec{k}^{T} \Upsilon-1 \vec{k}$. Now we can use standard theory to diagonalize this symmetric $2 \times 2$ matrix $\Upsilon$, with a rotation. This result is well known, but seems to be difficult to find in the literature. When the stiffness matrix is in diagonal form we will assume the diagonal entries are $k_{1}^{2}, k_{2}^{2}$ and $k_{3}^{2}$.

Next we turn to the invariants of the stiffness matrix $K$. That is we seek functions of the entries of $K$ which are invariant with respect to a rigid change in coordinates or rigid displacement. We can expect these to be symmetric polynomials in the diagonal elements of diagonalisation of $K$. The determinant of $K$ is clearly an invariant since it is an invariant under the group $S L(3)$ it must also be an invariant under the subgroup $S E(2)$. The determinant of the diagonal stiffness matrix is just the product of the stiffnesses, $\operatorname{det}(K)=k_{1}^{2} k_{2}^{2} k_{3}^{2}$. Notice that after a translation the bottom right $2 \times 2$ block of $K$ is unchanged. A rotation will change this block to,

$$
\Upsilon \longmapsto R \Upsilon R^{T}
$$

So rotational invariants of this block will be invariant under all of $S E(2)$. The invariants in question are $\operatorname{det}(\Upsilon)=k_{2}^{2} k_{3}^{2}$ and $\operatorname{Tr}(\Upsilon)=k_{2}^{2}+k_{3}^{2}$.

Next, a few examples will be considered, the first of these is a simple spring. This is an idealised component but the following two examples are a little more practical.

The simple spring is an idealisation of real springs, simple springs can be thought of as un-stretched springs with zero natural length. These elements are determined by lines in the plane. Given a line $\ell=$ $\left(n_{x}, n_{y},-d\right)^{T}$, the spring will only generate a wrench in the direction of the line, $\mathbf{w}=k \widetilde{L} \ell$, where $k$ is a constant The wrench produced by the simple spring is only in response to a small motion in the direction of the line, that is $\mathbf{w}^{T} \mathbf{s} \neq 0$ when $\mathbf{s}$ is a translation in the direction of $\ell$ but is zero for the orthogonal direction and infinitesimal rotations. This means that we can write the stiffness matrix as,

$$
K=\mathbf{w w}^{T}=k^{2} \widetilde{L} \ell \ell^{T} \widetilde{L} k^{2}\left(\begin{array}{ccc}
d^{2} & -d n_{y} & -d n_{x} \\
d n_{y} d & n_{y}^{2} & -n_{x} n_{y} \\
-d n_{x} & -n_{x} n_{y} & n_{x}^{2}
\end{array}\right),
$$

where $k^{2}$ is known and the stiffness constant of the spring. Usually this is stated the other way around the wrench produces a small motion of the spring.

If we think of lines $\ell$ as points in a projective plane $\mathbb{P}^{2}$ and stiffness matrices as points in a projective space $\mathbb{P}^{5}$ then the above is clearly a Veronese embedding. Hence the space of all possible simple springs is the degree 4, Veronese surface, see [2].

Next we consider simple beams. Again, here we can just restrict the stiffness matrix of the 3D beam, see [7]. The stiffness matrix of the slender planar beam aligned with the $x$-axis is thus,

$$
K=\left(\begin{array}{ccc}
\frac{E I_{y}}{l} & 0 & 0 \\
0 & \frac{E A}{l} & 0 \\
0 & 0 & \frac{12 E I_{y}}{l^{3}}
\end{array}\right)
$$


where $l$ is the length of the beam, $E$ is its Young's modulus, $A$ its cross-sectional area and $I_{y}$ the second moment of area. Note that, the stiffness matrix shown here is expressed in a coordinate frame whose origin is located at the centre of the beam and with the $x$-axis directed along the beam.

The space of these simple beams is a little more complicated than the simple springs. First we need to decide what to hold constant. For example, we could imagine that the beams that we have available are all made of the same material and have the same cross-section and length $l$. If this is the case then the space of all beams or rather the stiffness matrices of these beams is just the stiffness matrices which can be generated by rigid displacements of the diagonal stiffness matrix above. From the previous section it is clear that the diagonalisation of an arbitrary stiffness matrix is unique. Hence, the space of simple beams must be an embedding of the group manifold of $S E(2)$. This is well known to be isomorphic to the real projective space $\mathbb{P}^{3}$ with a single line deleted, see [1].

We can find several homogeneous equations satisfied by the stiffness matrices of simple beams. All that is needed is to find relations between the invariants of the matrices. These can be evaluated on the diagonal matrix given above,

$$
\begin{aligned}
\operatorname{det}(K) & =\frac{12 A E^{3} I_{y}^{2}}{l^{5}} \\
\operatorname{det}(\Upsilon) & =\frac{12 A E^{2} I_{y}}{l^{4}} \\
\operatorname{Tr}(\Upsilon) & =\left(\frac{E A}{l}+\frac{12 E I_{y}}{l^{3}}\right)=\frac{E}{l^{3}}\left(A l^{2}+12 I_{y}\right) .
\end{aligned}
$$

These invariants are of degree 3,2 and 1 respectively, in the elements of the stiffness matrix. So we can write a quadratic equation,

$$
12 A I_{y} l^{2} \operatorname{Tr}(\Upsilon)^{2}-\left(A l^{2}+12 I_{y}\right)^{2} \operatorname{det}(\Upsilon)=0,
$$

and a couple of cubic equations,

$$
12 A I_{y} l^{4} \operatorname{Tr}(\Upsilon)^{3}-\left(A l^{2}+12 I_{y}\right)^{3} \operatorname{det}(K)=0
$$

and

$$
I_{y} l^{2} \operatorname{Tr}(\Upsilon) \operatorname{det}(\Upsilon)-\left(A l^{2}+12 I_{y}\right) \operatorname{det}(K)=0
$$

and finally a degree 6 equation,

$$
12 A \operatorname{det}(K)^{2}-l^{2} I_{y} \operatorname{det}(\Upsilon)^{3}=0 .
$$

Presumably these four equations do not give a complete intersection in $\mathbb{P}^{5}$ but rather define a 3-dimensional rational variety.

If we assume that the simple beams can have different lengths then the space of all simple beams will be 4-dimensional. The equations defining this variety can be found by eliminating $l$ from the equations. Notice that the final degree 6 equation can be rearranged to isolate $l^{2}$ and the other three equations only contain $l^{2}$ or powers of $l^{2}$.

The final example here are stretched spring pairs. Consider the system of springs shown in Figure 1. The springs have natural length $l$ but in the equilibrium position illustrated are extended by an amount $e$. The springs are attached to the body at points a distance $2 d$ apart. The stiffness matrix of this arrangement can be found from the results in [6], 


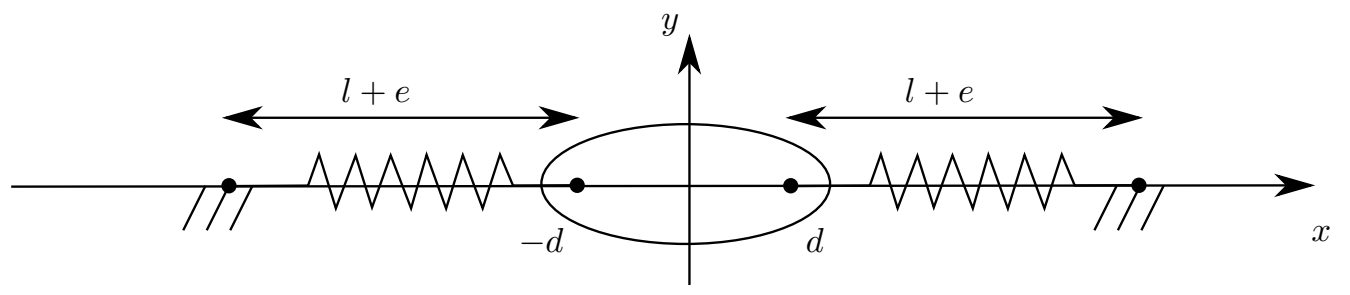

Figure 1: A Pair of Stretched Springs.

$$
K=2 \lambda e\left(\begin{array}{ccc}
d\left(1+\frac{d}{l+e}\right) & 0 & 0 \\
0 & \frac{1}{e} & 0 \\
0 & 0 & \frac{1}{l+e}
\end{array}\right),
$$

where $\lambda$ is the stiffness constant of the springs. It is, of course, possible to find homogeneous equations for the space of all stretched spring pairs using the same method we saw for the space of beams above.

\section{Synthesis}

Given a stiffness matrix, perhaps the specification of how one part of a machine needs to move with respect to another part, how can we select springs or beams to realise the given stiffness matrix? Unfortunately standard decomposition theorems of symmetric matrices don't apply here. The standard theory is based on eigenvalues and eigenvectors, these only make sense if the matrix represents a linear map from a space to itself or a map between Euclidean spaces. The matrix $K$ here is a map from twist space to wrench space, neither of these spaces is Euclidean.

It is important to note that if these systems of springs are combined in parallel the stiffness matrix of the result is simply the sum of the stiffness matrices of the component systems. Care must be taken however, to ensure that the stiffness matrices are expressed in the same coordinate system before they are added.

At the end of section 3 it was observed that the space of simple springs determine a Veronese surface in the $\mathbb{P}^{5}$ of all stiffness matrices. This is a two dimensional variety with degree $2^{2}=4$, [2]. By varying the modulus of stiffness of the springs we can produce all possible stiffness matrices lying on the line joining the stiffness matrices of the two simple springs. This is a chord or secant line to the surface. The closure of all such secant lines forms the secant variety to the surface. It is well known that the secant variety to the Veronese surface is a cubic hypersurface in $\mathbb{P}^{5}$ given by vanishing of a $3 \times 3$ determinant. Simple counting of dimensions would suggest that the secant variety to a surface should be 5-dimensional, however for the Veronese surface it is only 4-dimensional. This means that three simple springs will be needed to synthesise a general 2-D stiffness matrix. For singular stiffness matrices only one or two simple springs will be required.

Let $K$ be an arbitrary stiffness matrix, as we saw in section 3, there will be a rigid change of coordinates $M=\left(\begin{array}{cc}1 & 0 \\ J \vec{t} & R\end{array}\right)$, which diagonalises $K$

$$
M^{T} K M=\left(\begin{array}{ccc}
k_{1}^{2} & 0 & 0 \\
0 & k_{2}^{2} & 0 \\
0 & 0 & k_{3}^{2}
\end{array}\right) .
$$


In section 3 diagonalisation was effected using a displacement, a coordinate transformation just uses the inverse transformation.

Now consider the following three lines,

$$
\ell_{1}=\left(\begin{array}{c}
1 \\
0 \\
-1 / \sqrt{2}
\end{array}\right), \quad \ell_{2}=\left(\begin{array}{c}
-1 / 2 \\
\sqrt{3} / 2 \\
-1 / \sqrt{2}
\end{array}\right), \quad \ell_{3}=\left(\begin{array}{c}
-1 / 2 \\
-\sqrt{3} / 2 \\
-1 / \sqrt{2}
\end{array}\right)
$$

These are the edges of an equilateral triangle with the origin as centre. The corresponding wrenches are,

$$
\mathbf{w}_{1}=\frac{k_{1} \sqrt{3}}{\sqrt{2}}\left(\begin{array}{c}
-1 / \sqrt{2} \\
0 \\
1
\end{array}\right), \quad \mathbf{w}_{2}=\frac{k_{1} \sqrt{3}}{\sqrt{2}}\left(\begin{array}{c}
-1 / \sqrt{2} \\
-\sqrt{3} / 2 \\
-1 / 2
\end{array}\right), \quad \mathbf{w}_{3}=\frac{k_{1} \sqrt{3}}{\sqrt{2}}\left(\begin{array}{c}
-1 / \sqrt{2} \\
\sqrt{3} / 2 \\
-1 / 2
\end{array}\right) .
$$

Notice that all these springs have stiffness constant $k_{1} \sqrt{3} / \sqrt{2}$. A straightforward computation confirms that,

$$
\mathbf{w}_{1} \mathbf{w}_{1}^{T}+\mathbf{w}_{2} \mathbf{w}_{2}^{T}+\mathbf{w}_{3} \mathbf{w}_{3}^{T}=k_{1}^{2}\left(\begin{array}{ccc}
1 & 0 & 0 \\
0 & 1 & 0 \\
0 & 0 & 1
\end{array}\right)
$$

So we can write

$$
M^{T} K M=\Lambda U\left(\mathbf{w}_{1} \mathbf{w}_{1}^{T}+\mathbf{w}_{2} \mathbf{w}_{2}^{T}+\mathbf{w}_{3} \mathbf{w}_{3}^{T}\right) U^{T} \Lambda
$$

where $\Lambda=\left(\begin{array}{ccc}1 & 0 & 0 \\ 0 & k_{2} / k_{1} & 0 \\ 0 & 0 & k_{3} / k_{1}\end{array}\right)$ and $U$ is an arbitrary $3 \times 3$ orthogonal matrix, an element of $O(3)$. Now writing,

$$
\mathbf{v}_{i}=M^{-T} \Lambda U \mathbf{w}_{i}, \quad i=1,2,3
$$

we get the decomposition,

$$
K=\mathbf{v}_{1} \mathbf{v}_{1}^{T}+\mathbf{v}_{2} \mathbf{v}_{2}^{T}+\mathbf{v}_{3} \mathbf{v}_{3}^{T} .
$$

Moreover, this gives all possible such decompositions for a non-singular stiffness matrix $K$, different choices are given by different orthogonal matrices $U$.

In terms of simple springs this means that we can decompose a stiffness matrix $K$ into a system of three simple springs placed along the lines,

$$
\ell_{i}^{\prime}=\widetilde{L} \mathbf{v}_{i}, \quad i=1,2,3,
$$

Next we consider stretched spring pairs. For a general stiffness matrix in 2D we need at least two stretched string pairs. As above, we can assume that the stiffness matrix has been diagonalised by some rigid-body displacement $M$, so that we can write,

$$
K=\left(\begin{array}{ccc}
k_{1}^{2} & 0 & 0 \\
0 & k_{2}^{2} & 0 \\
0 & 0 & k_{3}^{2}
\end{array}\right)
$$

Assuming that we cannot change the stiffness of the material that the springs are made from means that we cannot use the secant variety to the space of spring pairs to synthesise the stiffness matrix. A possible strategy is to combine pairs with diagonal stiffness matrices. For example, suppose we rotate the pair by a 
quarter turn, such a pair can have a different extension $e_{2}$ and separation $d_{2}$ to the original pair $e_{1}$ and $d_{1}$. The stiffness matrix of such a perpendicular arrangement of pairs will give a stiffness matrix,

$$
K=2 \lambda e_{1}\left(\begin{array}{ccc}
d_{1}\left(1+\frac{d_{1}}{l+e_{1}}\right) & 0 & 0 \\
0 & \frac{1}{e_{1}} & 0 \\
0 & 0 & \frac{1}{l+e_{1}}
\end{array}\right)+2 \lambda e_{2}\left(\begin{array}{ccc}
d_{2}\left(1+\frac{d_{2}}{l+e_{2}}\right) & 0 & 0 \\
0 & \frac{1}{l+e_{2}} & 0 \\
0 & 0 & \frac{1}{e_{2}}
\end{array}\right) .
$$

Comparing this with the target stiffness gives 3 equations,

$$
\begin{aligned}
& k_{1}^{2}=2 \lambda\left(e_{1} d_{1}+e_{2} d_{2}+\frac{e_{1} d_{1}^{2}}{l+e_{1}}+\frac{e_{2} d_{2}^{2}}{l+e_{2}}\right), \\
& k_{2}^{2}=2 \lambda\left(1+\frac{e_{2}}{l+e_{2}}\right), \\
& k_{3}^{2}=2 \lambda\left(1+\frac{e_{1}}{l+e_{1}}\right) .
\end{aligned}
$$

The last two of these equations are easily solved,

$$
e_{1}=\left(\frac{k_{3}^{2}-2 \lambda}{4 \lambda-k_{3}^{2}}\right) l \quad \text { and } \quad e_{2}=\left(\frac{k_{2}^{2}-2 \lambda}{4 \lambda-k_{2}^{2}}\right) l .
$$

These result give limits on the stiffnesses that can be synthesised with such springs, if the extension is to be positive then we must have, $2 \lambda<k_{2}^{2}<4 \lambda$ and the same for $k_{3}^{2}$.

The first of the equations above would give many solutions for $d_{1}$ and $d_{2}$, so it seems reasonable to set $d_{1}=d_{2}=d$. This gives a quadratic equation for $d$,

$$
\left(\frac{e_{1}}{l+e_{1}}+\frac{e_{2}}{l+e_{2}}\right) d^{2}+\left(e_{1}+e_{2}\right) d-\frac{k_{1}^{2}}{2 \lambda}=0 .
$$

From the pattern of sign changes in the coefficients, this has one positive real solution for $d$, and this will give a stable equilibrium for the system.

An alternative solution might be to use a couple of identical spring pairs but move them relative to each other. Suppose we have two identical diagonal stiffness matrices and we subject them to either equal but opposite translations or equal but opposite rotations. The composite will be a diagonal stiffness matrix. For example, if we rotate the stiffness matrices of two stretched string pairs by $\theta$ and compare the combined system with the desired diagonal stiffness we get the following three equations,

$$
\begin{aligned}
& k_{1}^{2}=4 \lambda\left(e d+\frac{e d^{2}}{l+e}\right), \\
& k_{2}^{2}=4 \lambda\left(\cos ^{2} \theta+\frac{e}{l+e} \sin ^{2} \theta\right), \\
& k_{3}^{2}=4 \lambda\left(\sin ^{2} \theta+\frac{e}{l+e} \cos ^{2} \theta\right) .
\end{aligned}
$$

Adding the last two equations eliminates the angle so that we can solve for the extension,

$$
e=\left(\frac{k_{2}^{2}+k_{3}^{2}-4 \lambda}{8 \lambda-k_{2}^{2}-k_{3}^{2}}\right) l \text {. }
$$


Again, this gives limits on the stiffnesses which can be synthesised using this method with positive extensions $e$. Subtracting the last two equations gives a linear equation for $\cos (2 \theta)$,

$$
\cos (2 \theta)=\frac{k_{2}^{2}-k_{3}^{2}}{8 \lambda-k_{2}^{2}-k_{3}^{2}},
$$

since $l+e=(4 \lambda l) /\left(8 \lambda-k_{2}^{2}-k_{3}^{2}\right)$. Finally $d$ can be found as the unique positive real solution to the quadratic equation in $d$ given by the first equation above.

\section{Conclusions}

Similar techniques to those outlined above could be used to synthesis a desired stiffness from simple beams. We could also consider other compliant design elements. It was possible to find all possible designs using three simple springs, it would be useful to find all possible solutions for pairs of stretched springs and other stiffness elements.

It would also be useful to extend these ideas to 3 dimensional stiffness. However, in 3D a general stiffness matrix cannot necessarily be diagonalised with a rigid-body displacement. This makes the general problem of the synthesis of stiffness in 3D much more difficult.

\section{References}

[1] Bottema, O. and Roth, B., (1990), Theoretical Kinematics, Dover Publications, New York.

[2] J. Harris, (1992), Algebraic Geometry a first course, Springer Verlag, New York.

[3] S.Huang and J. Schimmels, (1998), "The bounds and realization of spatial stiffness achieved with simple springs connected in parallel”, IEEE Trans. Robot. Automat., 14(3):466-475.

[4] H. Lipkin and N. Ciblak, (1998), Application of stiffness decompositions to synthesis by springs, in Proc. ASME Design Tech. Conf., Atlanta, GA, pp. 13-16.

[5] R. Roberts, (2000), "Minimal realization of an arbitrary spatial stiffness matrix with a parallel connection of simple and complex springs", IEEE Trans. Robot. Automat., 16(5):603-608.

[6] J.M. Selig, (2000), The spatial stiffness matrix from simple stretched springs, in Proceedings of the IEEE International Conference on Robotics and Automation, San Francisco 2000 pp. 3314-3319.

[7] J.M. Selig and X.Ding, (2001), A screw theory of beams, in Proceedings of the IEEE/RSJ International Conference on Intelligent Robotics and Systems, Maui 2001 pp. 312-317.

[8] Selig, J.M. (2005), Geometric Fundamentals of Robotics, New York, Springer Verlag. 